\title{
Superstripes and Percolating Nanoscale- Striped Puddles in Heterostructures at Atomic Limit
}

\section{Antonio Bianconi}

Journal of Superconductivity and Novel Magnetism

Incorporating Novel Magnetism

ISSN 1557-1939

Volume 27

Number 4

J Supercond Nov Magn (2014)

27:909-912

DOI 10.1007/s10948-014-2516-1
Journal of

Superconductivity and Novel Magnetism

Volume $27 \cdot$ Number 4

April 2014

10948 - ISSN 1557-1939

27(4) $891-1110(2014)$

Springer

包 Springer 
Your article is protected by copyright and all rights are held exclusively by Springer Science +Business Media New York. This e-offprint is for personal use only and shall not be selfarchived in electronic repositories. If you wish to self-archive your article, please use the accepted manuscript version for posting on your own website. You may further deposit the accepted manuscript version in any repository, provided it is only made publicly available 12 months after official publication or later and provided acknowledgement is given to the original source of publication and a link is inserted to the published article on Springer's website. The link must be accompanied by the following text: "The final publication is available at link.springer.com". 


\title{
Superstripes and Percolating Nanoscale-Striped Puddles in Heterostructures at Atomic Limit
}

\author{
Antonio Bianconi
}

Published online: 13 March 2014

(C) Springer Science+Business Media New York 2014

\begin{abstract}
Lattice and electronic nanoscale phase separation in strongly correlated multiband systems confined in heterostructure at atomic limit called superstripes has been an object of the scientific debate at the international conference Superstripes 2013 focusing on "Quantum in Complex Matter: Superconductivity, Magnetism and Ferroelectricity" held in Ischia, Italy (May 27-June 1, 2013). The focus was on lattice granularity due to defects self-organization, lattice modulations at a critical misfit strain, and electronic phase separation in multiband Hubbard models near a 2.5 Lifshitz transition. The emerging superstripes scenario is a particular case of percolation superconductivity in networks of superconducting multicondensates superconducting puddles and their competition with phase-separated networks of nanoscale-striped magnetic puddles. This new emerging paradigm for high-Tc superconductor-layered oxides opens new perspectives for quantum electronics by controlling the complexity in functional oxides.
\end{abstract}

Keywords Nanoscale inhomogeneity · Transition metal oxides $\cdot$ Stripes $\cdot$ Quantum materials $\cdot$ Percolative superconductivity.

"Quantum in Complex Matter: Superconductivity, Magnetism and Ferroelectricity" has been the topic discussed at the Superstripes 2013 conference held in Ischia, Italy, from May 27 to June 1, 2013. The conference has been dedicated to a wide range of topics related to quantum electronic in

\footnotetext{
A. Bianconi $(\bowtie)$

RICMASS, Rome International Center for Materials Science Superstripes, Via dei Sabelli 119A, 00185 Rome, Italy

e-mail: antonio.bianconi@ricmass.eu
}

complex heterostructures at atomic limit. The heterostructures at the atomic limit and atomic layer include cuprates [1], diborides [2, 3], and iron-based superconductors [4-6] where the $\mathrm{CuO}_{2}$ atomic layer, the honeycomb boron atomic layer, and the atomic layers of $\mathrm{FeAs}_{4}$ or $\mathrm{FeSe}_{4}$ tetrahedral units are intercalated by variable spacer blocks. It is now feasible to tune the electronic structure by insertion of defects that get self-organized [6-13], under external pressure $[14,15]$, and lattice misfit strain [16] between the active atomic layers and the spacers and the gate voltage control [17]. Several authors have discussed electronic nanoscale phase separation in strongly correlated electronic systems [18-22]. There is a growing agreement that a generic feature of high temperature superconductors is phase separation in multiband Hubbard models in the proximity of a 2.5 Lifshitz transition in an interacting Fermi liquid [23] and that also cuprates show multiband or multicondensate superconductivity [24] like diborides [2] and iron-based superconductors [3]. Several sessions have been dedicated to the interplay of magnetic fluctuations and superconductivity [25-27] on inhomogeneous electronic crystals [28] and on cold Rydberg atoms for quantum simulation of exotic condensed matter interactions [29]. The emerging fundamental physics is a low-energy physics where electronic correlations, polaron formation [30-32], and defects selforganization determine and control minibands crossing the Fermi level where the Fermi level in each band is of the order of 50-100 meV [33].

The focus has been on the compelling evidence from many experiments that, in cuprates, there are different electronic states at the Fermi level and that they segregate in different spatial locations in the $\mathrm{CuO}_{2}$ plane [34-37]. The magnetic correlations in cuprates from the underdoped to overdoped phase determined by inelastic magnetic scattering and resonant inelastic $\mathrm{x}$-ray scattering are independent from 
the charge density waves determined by x-ray diffraction. There is clear evidence that high-temperature superconductivity appears to have broken lattice symmetry, and superstripes and multiple condensates appear in different positions in the k-space and in the real space. At the conference, it was clear that the proposal of mechanisms for high-temperature superconductivity based on a uniform electronic and lattice model have been falsified. The community is therefore looking now into very complex phase segregation landscapes at nanoscale where percolation superconductivity takes place in granular nanoscale matter as shown in Fig. 1.

Therefore, after many years of research on the mechanism of high-temperature superconductivity, following the discovery of Alex Müller in 1986 [38, 39], the scientific community has reached an agreement on the intrinsic heterogeneity of the $\mathrm{CuO}_{2}$ plane in superconducting $\mathrm{Cu}$ oxides on the basis of new experimental results probing the nanoscale structure of these complex oxides. The

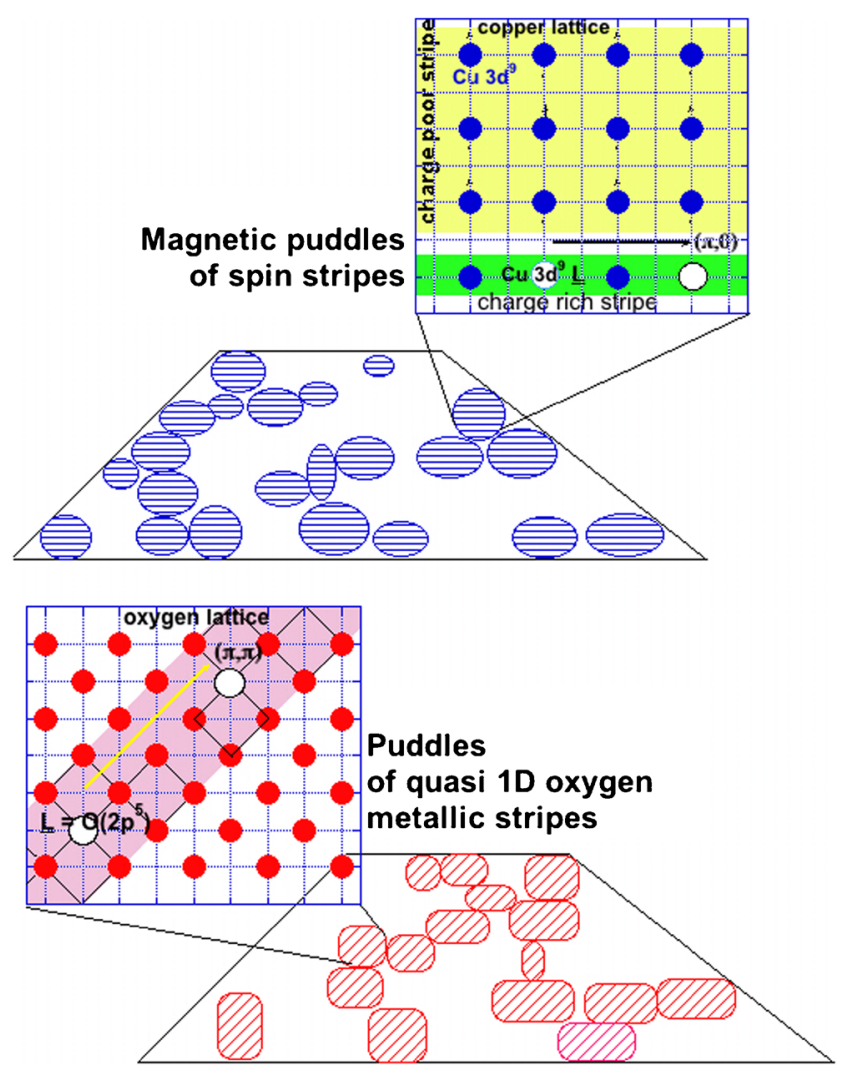

Fig. 1 Upper panel: A network of magnetic puddles (horizontal blue stripes) in $2 \mathrm{D}$ atomic $\mathrm{CuO}_{2}$ plane where the localized singlets $3 \mathrm{~d}^{9} \mathrm{~L}$ states in the $\mathrm{Cu}$ sites, induced by doping, form $1 \mathrm{D}$ stripes running in the direction of the $\mathrm{Cu}-\mathrm{O}-\mathrm{Cu}$ or $(0, \pi)$ antinodal direction. Lower panel: A network of different puddles (diagonal red stripes) in the 2D atomic $\mathrm{CuO}_{2}$ plane where the $3 \mathrm{~d}^{9} \mathrm{~L}$ states, induced by doping, form metallic $1 \mathrm{D}$ stripes running in the direction of the $\mathrm{O}-\mathrm{O}$ direction called $(\pi, \pi)$ nodal direction magnetic response of the copper oxide-layered perovskites, in scattering experiments probing magnetic fluctuations from underdoped to overdoped phase, is due to nanoscalestriped "magnetic puddles" of localized singlets located on the $\mathrm{Cu}$ sites forming nanoscale horizontal or vertical stripes as shown in the upper part of Fig. 1. These magnetic nanoscale puddles coexist in different spatial locations in the same $\mathrm{CuO}_{2}$ plane shown in the lower part of the figure with other different puddles shown in the lower panel of Fig. 1 made by $\mathrm{O}(2 \mathrm{p})$ holes in the oxygen ion sublattice running in quasi $1 \mathrm{D}$ metallic stripes in the diagonal lattice direction.

This electronic and lattice complexity should be assigned to a complexity of interactions involving polaron selforganization, lattice modulations due to lattice parameter misfit strain, and self-organization of dopants in the spacer layers.

This scenario supports the presence of two electronic components at the Fermi level of cuprates: localized multielectron configurations $3 \mathrm{~d}^{9} \mathrm{~L}$ detected in April 1987 [40, 41] by measuring many-body final states in XANES spectroscopy $[42,43]$ which can give either localized singlets on the $\mathrm{Cu}$ sublattice and itinerant oxygen $2 \mathrm{p}$ holes in the oxygen sublattice.

The superconducting nanoscale portions are confined in nanoscale puddles or at their interface in these heterostructures at the atomic limit. This scenario called "superstripes" in $2000[44,45]$ is a particular case of percolation superconductivity at a nanoscale proposed by several authors [46-49] where the disorder could favor the increase of critical temperature in the percolation regime where scale-free superconducting networks are formed [50, 51].

Finally, a novel scenario of percolation superconductivity made by nanoscale puddles in heterostructures at the atomic limit, involving granular nanoscale low-dimensional multicondensates, has emerged at the Superstripes 2013 in Ischia. A similar scenario has been reported to appear also in iron-based superconductors where the superstripes seem to be formed on the domain walls of magnetic puddles, and it is opening a new perspective for the design of new high-temperature superconductors.

\section{References}

1. Bianconi, A.: Solid State Comm. 89, 933-936 (1994). doi:10. 1016/0038-1098(94)90354-9

2. Bussmann-Holder, A., Bianconi, A.: Raising the diboride superconductor transition temperature using quantum interference effects. Phys. Rev. B 67, 132509 (2003). doi:10.1103/physrevb. 67.132509

3. Innocenti, D., Poccia, N., Ricci, A., Valletta, A., Caprara, S., Perali, A., Bianconi, A.: Phys. Rev. B 82, 184528 (2010). doi:10.1103/physrevb.82.184528 
4. Ricci, A., Poccia, N., Joseph, B., Arrighetti, G., Barba, L., Plaisier, J., Campi, G., Mizuguchi, Y., Takeya, H., Takano, Y., Saini, N.L., Bianconi, A.: Supercond. Sci. Tech. 24, 082002+ (2011). http:// iopscience.iop.org/0953-2048/24/8/082002/

5. Ricci, A., Joseph, B., Poccia, N., Campi, G., Saini, N.L., Bianconi, A.: Temperature dependence of $\sqrt{ } 2 \mathrm{x} \sqrt{ } 2$ phase in superconducting $\mathrm{K}_{0.8} \mathrm{Fe}_{1.6} \mathrm{Se}_{2}$ single crystal. J. Supercond. Novel Magn. 1-5 (2013). doi:10.1007/s10948-013-2426-7

6. Joseph, B.: Fe-As bond-dynamics revealed from the arsenic K-edge EXAFS studies of NdFeAsO1-x iron-pnictide superconductors. J. Supercond. Novel Magn. 1-3 (2013). doi:10.1007/ s10948-013-2421-z

7. Geballe, T.H., Marezio, M.: Physica C 469, 680 (2009)

8. Poccia, N., Fratini, M., Ricci, A., Campi, G., Barba, L., VittoriniOrgeas, A., Bianconi, G., Aeppli, G., Bianconi, A.: Nat. Mater. 10, 733-736 (2011). doi:10.1038/nmat3088

9. Campi, G., Ricci, A., Poccia, N., Bianconi, A.: Imaging spatial ordering of the oxygen chains in $\mathrm{YBa}_{2} \mathrm{Cu}_{3} \mathrm{O}_{6+y}$ at the insulatorto-metal transition. J. Supercond. Novel Magn. 1-4 (2013). doi:10.1007/s10948-013-2434-7

10. Zaanen, J.: Nature 466, 825 (2010)

11. de Mello, E.V.L.: EPL (Europhys. Lett.) 98, 57008 (2012)

12. de Mello, E.V.L.: Ibidem EPL (Europhys. Lett.) 99, 37003 (2012)

13. Littlewood, P.: Nat. Mater. 10, 726 (2011)

14. Bendele, M., Pomjakushina, E., Conder, K., Khasanov, R., Keller, H.: Pressure effects in the iron chalcogenides. J. Supercond. Novel Magn. 1-4 (2013). doi:10.1007/s10948-013-2419-6

15. Aoki, H., Kariyado, T.: Pressure effects and orbital characters in cuprate and carbon-based superconductors. J. Supercond. Novel Magn. 1-7 (2013). doi:10.1007/s10948-013-2425-8

16. Agrestini, S., Saini, N.L., Bianconi, G., Bianconi, A.: J. Phy. Math. Gen. 36, 9133-9142 (2003). doi:10.1088/0305-4470/36/35/302

17. Ye, J.T., Zhang, Y.J., Yoshida, M., Saito, Y., Iwasa, Y.: Fieldinduced superconductivity in $\mathrm{MoS}_{2}$. J. Supercond. Novel Magn. 1-5 (2013). doi:10.1007/s10948-013-2422-y

18. Kapcia, K.: Metastability and phase separation in a simple model of a superconductor with extremely short coherence length. J. Supercond. Novel Magn. 1-5 (2013). doi:10.1007/s10948013-2409-8

19. Seibold, G., Bünemann, J., Lorenzana, J.: Time-dependent Gutzwiller approximation: interplay with phonons. J. Supercond. Novel Magn. 1-3 (2013). doi:10.1007/s10948-013-2412-0

20. Mukhin, S.I.: Euclidian crystals in many-body systems: breakdown of Goldstone's theorem. J. Supercond. Novel Magn., 1-6 (2013). doi:10.1007/s 10948-013-2416-9

21. García Saravia Ortíz de Montellano, A., Mustre de León, J.: Characterization of an electronic excitation on a Peierls-Hubbard Hamiltonian for a small cluster. J. Supercond. Novel Magn. 1-4 (2013). doi:10.1007/s10948-013-2424-9

22. Krinitsyn, A., Nikolaev, S., Ovchinnikov, S.: Cluster size and shape effect on the electronic structure of the Hubbard model within the norm-conserving cluster perturbation theory. J. Supercond. Novel Magn. 1-9 (2013). doi:10.1007/s 10948-013-2418-7

23. Kugel, K., Rakhmanov, A., Sboychakov, A., Poccia, N., Bianconi, A.: Model for phase separation controlled by doping and the internal chemical pressure in different cuprate superconductors. Phys. Rev. B 78, 165124+ (2008). doi:10.1103/physrevb.78.165124

24. Nikšić, G., Kupčić, I., Barišić, O.S., Sunko, D.K., Barišić, S.: Multiband responses in high-T c cuprate superconductors. J. Supercond. Novel Magn. 1-7 (2013). doi:10.1007/s10948013-2420-0

25. Sugai, S., Takayanagi, Y., Nohara, J., Shiozaki, R., Hayamizu, N., Muroi, T., Hosokawa, T., Suzuki, H., Sone, Y., Mabuchi, H., Takenaka, K., Okazaki, K.: Stripe-induced high-temperature superconductivity in cuprates. J. Supercond. Novel Magn. 1-4 (2013). doi:10.1007/s10948-013-2415-x
26. Fujiwara, N., Iimura, S., Matsuishi, S., Hosono, H., Yamakawa, Y., Kontani, H.: NMR study on a pnictide superconductor LaFeAsO1-x $h$ in a H-overdoped regime: revival of antiferromagnetic fluctuations. J. Supercond. Novel Magn. 1-4 (2013). doi:10.1007/s10948-013-2413-z

27. Rudnev, I., Osipov, M.: Local study of magnetic structures in high-temperature superconducting composites. J. Supercond. Novel Magn., 1-4 (2013). doi:10.1007/s10948-013-2417-8

28. Hague, J.P., Downes, S., MacCormick, C., Kornilovitch, P.E.: Cold rydberg atoms for quantum simulation of exotic condensed matter interactions. J. Supercond. Novel Magn. 1-4 (2013). doi:10.1007/s10948-013-2414-y

29. Brazovskii, S., Kirova, N.: Excitonic mechanism of local phase transformations by optical pumping. J. Supercond. Novel Magn. 1-5 (2013). doi:10.1007/s10948-013-2432-9

30. Bianconi, A., Missori, M., Oyanagi, H., Yamaguchi, H., Nishiara, Y., Della Longa, S.: EPL (Europhys. Lett.) 31, 411-415 (1995). http://iopscience.iop.org/0295-5075/31/7/012

31. Bussmann-Holder, A., Keller, H., Khasanov, R., Simon, A., Bianconi, A., Bishop, A.R.: New J. Phys. 093009, 13 (2011). doi:10.1088/1367-2630/13/9/093009

32. Menushenkov, A.P., Kuznetsov, A.V., Chernikov, R.V., Ivanov, A.A., Sidorov, V.V., Klementiev, K.V.: Low temperature anharmonicity and superconductivity in cuprates. J. Supercond. Novel Magn. 1-4 (2013). doi:10.1007/s10948-013-2411-1

33. Jarlborg, T., Bianconi, A.: Fermi surface reconstruction of superoxygenated $\mathrm{La} 2 \mathrm{CuO} 4$ superconductors with ordered oxygen interstitials. Phys. Rev. B 87, 054514 (2013). doi:10.1103/physrevb. 87.054514

34. Hucker, M., Zimmermann, M.V., Xu, Z.J., Wen, J.S., Gu, G.D., Tranquada, J.M.: Phys. Rev. B 87, 014501 (2013)

35. Blackburn, E., Chang, J., Hucker, M., Holmes, A.T., Christensen, N.B., Liang, R., Bonn, D.A., Hardy, W.N., Rutt, U., Gutowski, O., Zimmermann, M.v., Forgan, E.M., Hayden, S.M.: Phys. Rev. Lett. 110, 137004 (2013)

36. Blanco-Canosa, S., Frano, A., Loew, T., Lu, Y., Porras, J., Ghiringhelli, G., Minola, M., Mazzoli, C., Braicovich, L., Schierle, E., Weschke, E., Le Tacon, M., Keimer, B.: Phys. Rev. Lett. 110, 187001 (2013)

37. Udby, L., et al.: Phys. Rev. Lett. 111, 227001 (2013). doi:10.1103/ PhysRevLett.111.227001

38. Müller, K.A., Bednorz, J.G.: A road towards high-temperature superconductivity. Phys. Scr. 1987, 198723 In: Proceedings of the Symposium on High $\mathrm{T}_{c}$ Superconductivity at the 7th General Conference of the Condensed Matter Division of the European Physical Society, Pisa, Italy, April 8 1987. http://m. iopscience.iop.org/1402-4896/1987/T19A/003?rel=sem\&relno=1. (1987). doi:10.1088/0031-8949/1987/T19A/003

39. Müller, K.A.: Phys. C 185-189, 3-10. Proc. 3rd International Conference. on Materials and Mechanisms of Superconductivity, High Temperature Superconductors, Kanazawa Japan, 22-26 July (1991)

40. Bianconi, A., Congiu Castellano, A., De Santis, M., Rudolf, P., Lagarde, P., Flank, A.M., Marcelli, A.: L2,3 XANES of the high $\mathrm{Tc}$ superconductor $\mathrm{YBa} 2 \mathrm{Cu} 3 \mathrm{O} 7$ with variable oxygen content. In: Proceedings of the Symposium on High $\mathrm{T}_{c}$ Superconductivity at the 7th General Conference of the Condensed Matter Division of the European Physical Society, Pisa, Italy, 8 April, 1987. http://adsabs.harvard.edu/abs/1987eps..symp..... (1987)

41. Bianconi, A., Congiu-Castellano, A., De Santis, M., Rudolf, P., Lagarde, P., Flank, A.M., Marcelli, A.: $\mathrm{L}_{2,3}$ XANES of the high $\mathrm{T}_{c}$ superconductor $\mathrm{YBa}_{2} \mathrm{Cu}_{3} \mathrm{O}_{7}$ with variable oxygen content. Solid State Comm. 63, 1009 (1987). doi:10.1016/00381098(87)90650-8 
42. Bianconi, A., Garcia, J., Benfatto, M.: XANES in condensed systems in synchrotron radiation in chemistry and biology I. In: Mandelkow, E. (ed.) Topics in Current Chemistry, vol. 145, pp. 29-67. Springer, Heidelberg (1988)

43. Della Longa, S., Soldatov, A., Pompa, M., et al.: Comput. Mater. Sci 4, 199-210 (1995)

44. Bianconi, A.: Int. J. Mod. Phys. B 14, 3289-3297 (2000). doi:10.1142/S0217979200003769

45. Bianconi, A., Di Castro, D., Bianconi, G., Pifferi, A., Saini, N.L., Chou, F.C., Johnston, D.C., Colapietro, M.: Phys. C Supercond. 341, 1719 (2000). doi:10.1016/s0921-4534(00)00950-3

46. Kresin, V.Z., Friedel, J.: EPL (Europhys. Lett.) 93, 13002 (2011). doi:10.1209/0295-5075/93/13002
47. Ovchinnikov, Y.N., Kresin, V.Z.: Phys. Rev. B 85, 064518 (2012). doi:10.1103/PhysRevB.85.064518

48. Yukalov, V.I., Yukalova, E.P.: Statistical theory of materials with nanoscale phase separation. J. Supercond. Novel Magn. 1-6 (2013). doi:10.1007/s10948-013-2410-2

49. Phillips, J.C.: Ineluctable complexity of high temperature superconductivity elucidated. J. Supercond. Novel Magn. 27, 345-347 (2014). doi:10.1007/s10948-013-2308-z

50. Bianconi, G.: Superconductor-insulator transition on annealed complex networks. Phys. Rev. E 85, 061113+ (2012). doi:10.1103/physreve.85.061113

51. Bianconi, A.: Quantum materials: shape resonances in superstripes. Nat. Phys. 9, 536-537 (2013). doi:10.1038/nphys2738 